\title{
Failure Rate Minimization with Multiple Function Unit Scheduling for Heterogeneous WSNs
}

\author{
Meikang Qiu ${ }^{\dagger} \quad$ Jing Deng ${ }^{\ddagger} \quad$ Edwin H.-M. Sha* \\ $\dagger$ Dept. of Electrical Engineering, University of New Orleans, New Orleans, LA 70148, USA \\ $\ddagger$ Dept. of Computer Science, University of North Carolina at Greensboro, Greensboro, NC 27402, USA \\ * Dept. of Computer Science, University of Texas at Dallas, TX 75083, USA
}

\begin{abstract}
Failure-Rate Minimization is becoming one of the major design issues in wireless sensor network (WSN) architecture due to multiple available Functional-units (FUs). There is a tradeoff between reliability and performance, such as timing constraint. This paper studies how to minimize the total failure rate while satisfying performance requirement for WSN applications. Two novel algorithms are proposed to solve the FRMFS (Failure Rate Minimization with FU Scheduling) problem. We use these FU scheduling algorithms to minimize system failure rate without sacrificing performance. Our results show that the average improvement on failure-rate reduction is significant with the use of our algorithms.
\end{abstract}

Index Terms-Heterogeneous, scheduling, failure rate, multi-FU, minimization

\section{INTRODUCTION}

Wireless sensor networks (WSNs) have a wide variety of applications, for example: microclimate monitoring [1], [2], plant physiology [3], animal behavior [4]-[6], structural monitoring [2], [7], [8] and condition-based maintenance. Reliability of distributed micro sensor nodes is an important issue in the design of sensor networks.

Heterogeneity is the trend of today's WSNs. With more and more sensor types or Functional-units (FUs) available, it is challenging to take full advantage of these FUs while satisfying the timing constraints in WSN design. The same function can be executed by different sensor type or FU types. Therefore, the same type of operations can be processed by heterogeneous sensors or FUs with different costs or failure rates. This paper focuses on how to reduce the total system failure rate of a WSN composed of heterogeneous sensors (FUs).

According to the failure rate model proposed by Srinivasan and Jha [9], the failure rate of a system is defined as the rate of failure of a system or component during during the time of executing a DFG (Data Flow Graph). A DFG is a node-weighted and edge-weighted directed graph, where each node represents a task, and each edge defines the precedence relations among nodes. In order to minimize the system failure rate, we need to minimize the sum of failure rates of all the nodes in the system. In other words, we need

This work is partially supported by NSF CCR-0309461, NSF IIS0513669, HK CERG B-Q60B, NSFC 60728206, Louisiana EPSCoR Pfund LBOR0049PR00C and BoR RCS grant LEQSF (2005-08)-RD-A-43. to find a schedule such that the timing constraint is satisfied and the sum of failure rates of all nodes is minimized.

Combining the consideration of failure rate [?], [9], [10] and performance, we design in this paper two algorithms to solve the FRMFS (Failure Rate Minimization with $F U$ Scheduling) problem, i.e., how to minimize total failure rate while satisfying timing constraint for WSN applications. Our experimental results show that these algorithms achieve significant reductions on an average in total failure rate compared with the previous works. For example, with 3 FUs, compared with the TBFT algorithm in [9], our algorithm FUSCh2 shows an average $21.7 \%$ reduction in total failure rate.

The paper is organized as follows: we describe necessary background in Section II. Our proposed algorithms are presented in Section III. In Section IV, experimental results are shown and discussed. Concluding remarks are provided in Section V.

\section{BASIC CONCEPTS AND MODELS}

In this section, we introduce some basic concepts that will be used throughout this paper. First we present the system failure rate model. Then, we describe the FUs scheduling problem with the help of an example.

\section{A. The Failure Rate Model}

We use the failure rate model proposed by Srinivasan and Jha [9]. Consider a heterogeneous system with $M$ FUs available, $\left\{F_{1}, F_{2}, \cdots, F_{M}\right\}$, and a DFG containing $N$ nodes, $\left\{u_{1}, u_{2}, \cdots, u_{N}\right\}$. Let $t_{j}(i)$ be the execution time of node $u_{i}$ for FU $F_{j}$. Let $f_{j}$ be the failure rate of $F U F_{j}$. Then the failure rate of node $u_{i}$ for $F U F_{j}$ is defined as $t_{j}(i) \cdot f_{j}$. Let $x_{i j}$ be a binary number that denotes whether FU $F_{j}$ is scheduled to node $u_{i}$ or not (it equals 1 if $F U F_{j}$ is assigned to $u_{i}$; otherwise it equals 0 ).

Define the un-failure rate of a system as the probability that the system will not fail during the time of executing a DFG. The probability of a system not failing during the time of processing a DFG is:

$$
\operatorname{Pr}=\prod_{1 \leq j \leq M, 1 \leq i \leq N}\left(1-f_{j}\right)^{x_{i j} t_{j}(i)} .
$$


When $f_{j} \ll 1$, we can approximate

$$
\left(1-f_{j}\right)^{x_{i j} t_{j}(i)} \approx 1-f_{j} x_{i j} t_{j}(i) \approx e^{-f_{j} x_{i j} t_{j}(i)} .
$$

Therefore, we have

$$
\operatorname{Pr} \approx \prod\left(e^{-f_{j} x_{i j} t_{j}(i)}\right)
$$

when $f_{j} \ll 1[11]$.

Thus, in order to maximize $\mathrm{Pr}$, we need to minimize $\sum\left(f_{j} x_{i j} t_{j}(i)\right)$, which is called as failure rate of a system In other words, we need to find a schedule such that the timing constraint is satisfied and the sum of failure rates of all nodes is minimized, which is needed to avoid system failure as much as possible.

\section{B. Heterogeneous $F U$ schedule problem}

We define the heterogeneous FU scheduling problem as follows: given a heterogeneous system with $M$ FUs, $F=F_{1}, \cdots, F_{i}, \cdots, F_{M}$, a DAG $G=\left\langle V, E_{d}\right\rangle$ where $V=\left\langle u_{1}, \cdots, u_{i}, \cdots, u_{N}\right\rangle$ is a set of nodes, with each node representing a task, $E_{d} \subseteq \mathrm{V} \cdot \mathrm{V}$ is a set of edges representing dependencies relations among nodes in $V$. $\mathrm{T}\left(\mathrm{u}_{\mathrm{k}}\right)=\mathrm{t}_{1}(\mathrm{k}), \cdots, \mathrm{t}_{\mathrm{i}}(\mathrm{k}), \cdots, \mathrm{t}_{M}(\mathrm{k})$, where $\mathrm{t}_{\mathrm{i}}(\mathrm{k})$ denoted the computation time of $u_{k}$ on $F_{i}$, and a time constraint $\mathrm{L}$, find a task schedule for $\mathrm{G}$ such that the failure rate is minimized within L.

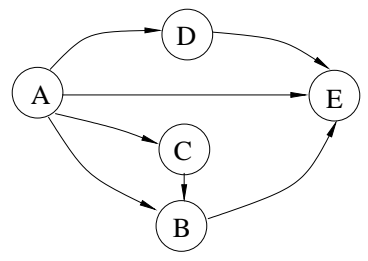

(a)

\begin{tabular}{|c|c|c|c|c|}
\hline \multirow{2}{*}{ Node } & \multicolumn{2}{|c|}{ F1 } & \multicolumn{2}{c|}{ F2 } \\
\cline { 2 - 5 } & T1 & E1 & T2 & E2 \\
\hline A & 2 & 6 & 1 & 15 \\
\hline B & 2 & 6 & 1 & 15 \\
\hline C & 4 & 12 & 3 & 27 \\
\hline D & 3 & 9 & 3 & 24 \\
\hline E & 3 & 9 & 2 & 15 \\
\hline
\end{tabular}

(b)
Fig. 1. (a) A DAG. (b) Execution times and failure rate of FUs.

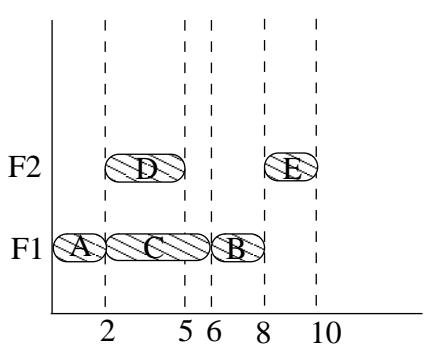

(a)

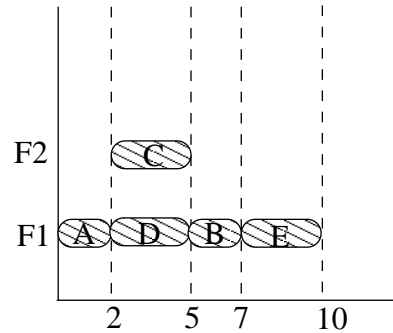

(b)
Fig. 2. (a) Schedule 1. (b) Schedule 2.

An example is shown in Fig. 1 and Fig. 2. Assume there is a heterogeneous system that consists of two heterogeneous
FUs, F1 and F2. An exemplary DAG is shown in Fig. 1(a). The given time constraint for the DAG to be executed is 10 time units. The execution time and failure rate of each node for different FUs are shown in Fig. 1(b). For the convenience of computation and presentation, we use an integer (enlarged 1000 times) to represent the failure rate of each node. In Fig. 1(b), $T_{i}$ denotes the execution time and $E_{i}$ denotes the first part of the failure rate $E_{i j}$, which is the failure rate for $u_{i}$ to be scheduled on $F_{j}$. Two schedules for the DAG are shown in Fig. 2. In Schedule 1, the schedule length is 10 time units and its system failure rate is 63. In Schedule 2, the schedule length is 10 time units and its system failure rate is 57 . Both schedules satisfy the time constraint while the latter has a lower failure rate. This example shows that different task schedules will produce different system failure rate.

\section{THE ALgORITHMS}

In this section, two algorithms, FUSSch1 and FU_Sch2 are designed to solve the FRMFS (Failure Rate Minimization with FU Scheduling) problem, i.e., how to obtain the minimum total system failure rate without sacrificing performance, based on multi-FU scheduling.

Scheduling problems with time and resource constraints are well-known to be NP complete. We are going to solve a scheduling problem with time and resource constraints in a heterogeneous system and minimize the failure rate of the system at the same time. Therefore, our problem is also NP complete. In this section, two novel algorithms, $F U \_S c h 1$ and $F U_{-} S c h 2$, are developed to solve this problem. $F U S c h 1$ uses a bipartite matching strategy based on ALAP (As Late As Possible) scheduling and FU_Sch2 uses a progressive relaxation strategy based on ALAP scheduling. Some symbols used in our algorithms are listed in Table I.

\begin{tabular}{|c||c|}
\hline \multicolumn{1}{|c|}{ Symbol } & Meaning \\
\hline \hline Min & Minimum failure rate for the current node \\
\hline EST $_{i}$ & Earliest starting time for node $i$ \\
\hline $\mathrm{LST}_{i}$ & Latest starting time for node $i$ \\
\hline $\mathrm{FT}_{i}$ & Finish time of node $i$ \\
\hline $\mathrm{SL}_{j}$ & Schedule length for $\mathrm{FU}_{j}$ \\
\hline $\mathrm{X}_{i}$ & The FU that node $i$ is scheduled on \\
\hline $\mathrm{E}_{\text {total }}$ & Overall failure rate for the system \\
\hline
\end{tabular}

TABLE I

SYMBOLS IN THE FU SCHEDULING ALGORITHMS

\section{A. FU Scheduling with Bipartite Matching}

$F U \_S c h 1$ is designed to use the bipartite matching strategy to schedule tasks. The idea is: first, use the ALAP scheduling, which minimizes the schedule length, to get the Tatest starting time for every node. Then construct a bipartite matching graph with the nodes in the ready list in one set and all FUs in the other set. Then reschedule nodes based on the minimum failure-rate bipartite matching. This algorithm is shown in Algorithm III.1 $V_{1}$ and $V_{2}$ in the algorithm represent the two sets used in the bipartite matching.

This algorithm first schedules all nodes using the ALAP scheduling. Based on the schedule, we use the bipartite 


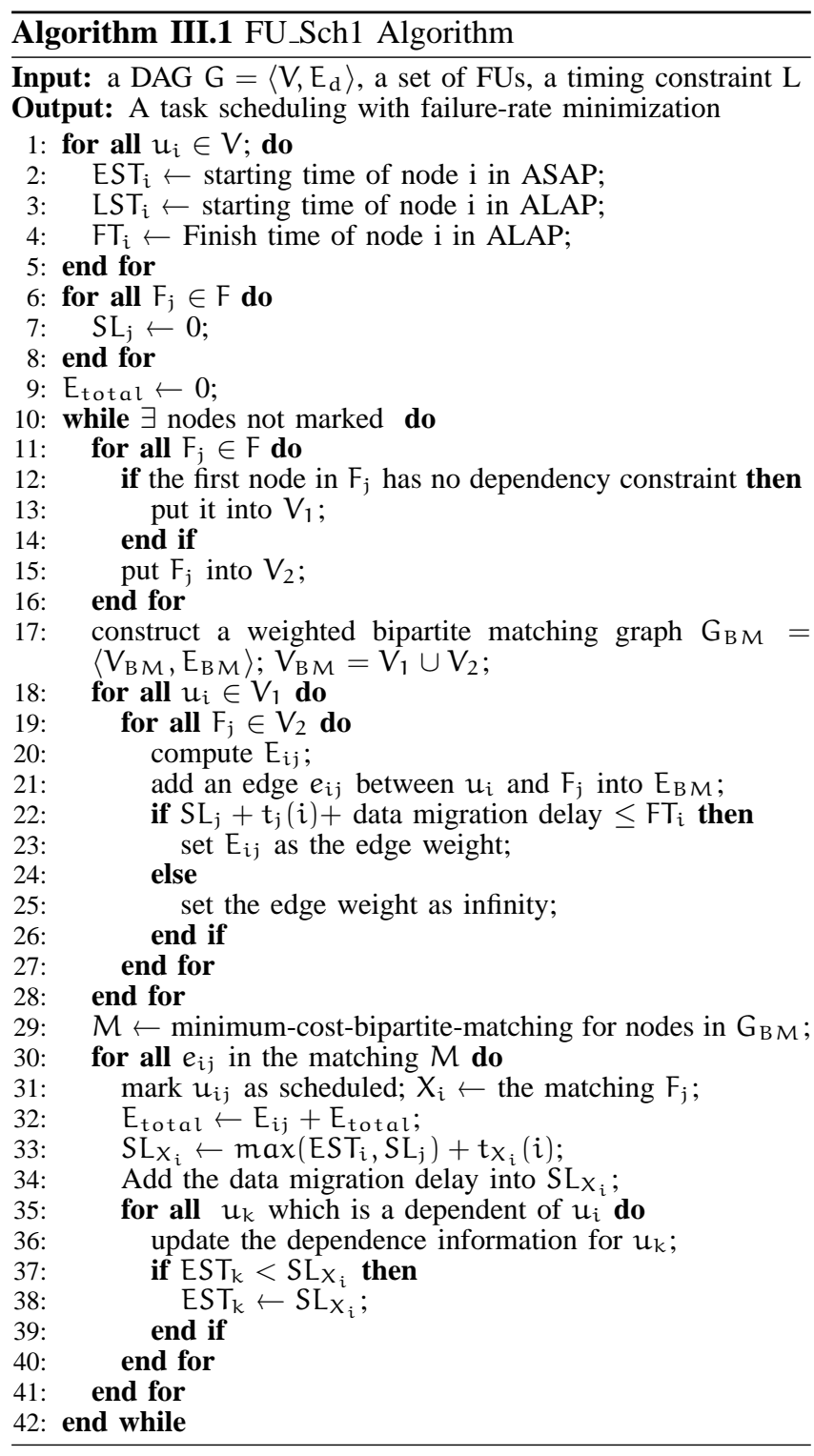

matching to schedule nodes. For each FU, among those nodes not marked by matching, the node with the earliest start time is considered: if it has no dependency constraint at that time, it's inserted into the ready list. A bipartite matching graph is constructed as follows: all nodes from the ready list are on one side, denoted by a set $V_{1}$, and all FUs are on the other side, denoted by a set $V_{2}$. Each node, $u_{i}$, in $V_{1}$ has an edge connected with each $F U, F_{j}$, in $V_{2}$. If the schedule length of the FU plus the node's computation time is less than the finish time of the node in ALAP, the edge weight is set to the failure rate $E_{i j}$. Otherwise, the edge weight is set to be infinity. After constructing the graph, call the minimum-cost-bipartitematching function to get a minimum cost bipartite matching. Since the edge weight is set to the failure rate, the matching produced by the function minimizes the reliability cost in

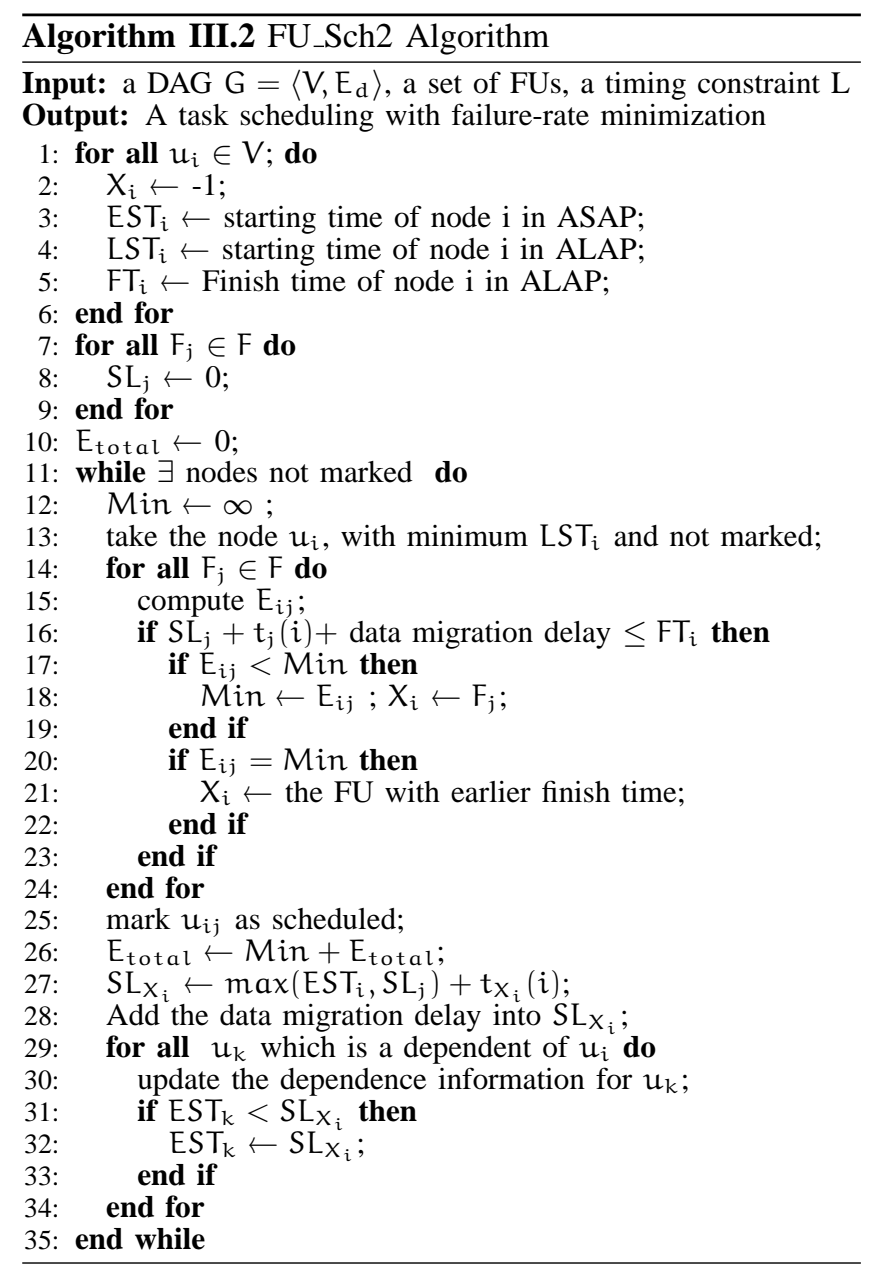

each scheduling step. After a match is found, schedule the tasks on the corresponding FUs, mark the node and update their descendants' information, i.e. dependence constraints and earliest starting time, recursively. Repeat this process until there is no more node to be rescheduled.

Because the FU selection for a node is limited by the finish time in the ALAP, the node can at least be scheduled on the same FU as ALAP. Thus, as long as there is an ALAP schedule for the graph, this algorithm won't fail to produce a schedule for all the tasks. As the failure rate is the matching factor in the bipartite matching, the failure-rate minimization is improved over the list scheduling algorithm.

\section{B. FU Scheduling with Progressive Relaxation}

$F U_{-} S c h 2$ progressively improves the reliability based on the schedule obtained by the ALAP scheduling. The idea is to reschedule each node to reduce the system failure rate as much as possible. It is shown in Algorithm III.2. After the initialization, this algorithm first obtains an ALAP schedule for all the tasks in the graph. Then repeat the following steps until all nodes are marked: among all nodes that are not marked, take the node with earliest starting time and reschedule it to a FU such that the system failure 


\begin{tabular}{|c|c|c|c|c|c|c|c|c|c|}
\hline \multicolumn{10}{|c|}{ Four Methods Comparison with 3 FUs } \\
\hline \multirow[t]{2}{*}{ Bench. } & \multirow[t]{2}{*}{$\mathrm{N}$. } & M1 & M2 & \multicolumn{3}{|c|}{ M3 } & \multicolumn{3}{|c|}{ M4 } \\
\hline & & $\begin{array}{c}\mathrm{E} \\
(\mu \mathrm{J})\end{array}$ & $\begin{array}{c}\mathrm{E} \\
(\mu \mathrm{J})\end{array}$ & $\begin{array}{c}\mathrm{E} \\
(\mu \mathrm{J})\end{array}$ & $\begin{array}{c}\% \text { M1 } \\
(\%)\end{array}$ & $\begin{array}{c}\% \text { M2 } \\
(\%)\end{array}$ & $\begin{array}{c}\mathrm{E} \\
(\mu \mathrm{J})\end{array}$ & $\begin{array}{c}\% \text { M1 } \\
(\%)\end{array}$ & $\begin{array}{c}\% \text { M2 } \\
(\%)\end{array}$ \\
\hline WDF(1) & 4 & 625 & 550 & 446 & 28.6 & 18.9 & 431 & 31.0 & 21.6 \\
\hline WDF(2) & 12 & 1862 & 1649 & 1335 & 28.3 & 19.0 & 1284 & 31.0 & 22.1 \\
\hline IIR & 16 & 2456 & 2135 & 1751 & 28.7 & 18.0 & 1678 & 31.7 & 21.4 \\
\hline DPCM & 16 & 2548 & 2267 & 1837 & 27.9 & 19.0 & 1762 & 30.8 & 22.3 \\
\hline $2 \mathrm{D}(1)$ & 34 & 5402 & 4732 & 3825 & 28.6 & 18.5 & 3725 & 31.0 & 21.3 \\
\hline $2 \mathrm{D}(2)$ & 4 & 665 & 591 & 478 & 28.1 & 19.1 & 461 & 30.7 & 22.0 \\
\hline MDFG1 & 8 & 1321 & 1153 & 942 & 28.7 & 18.3 & 902 & 31.7 & 21.8 \\
\hline MDFG2 & 8 & 1487 & 1325 & 1075 & 27.7 & 18.9 & 1034 & 30.5 & 22.0 \\
\hline Floyd & 16 & 2624 & 2259 & 1872 & 28.7 & 17.1 & 1801 & 31.4 & 20.3 \\
\hline All-pole & 29 & 4475 & 3946 & 3211 & 28.2 & 18.6 & 3082 & 31.1 & 21.9 \\
\hline & 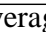 & Reduc & n (\%) & & 28.4 & 18.5 & & 31.1 & 21.7 \\
\hline
\end{tabular}

TABLE II

THE COMPARISON OF TOTAL FAILURE RATE WITH FOUR METHODS ON VARIOUS BENCHMARKS WHEN THE TIME CONSTRAINT IS 1000.

\begin{tabular}{|c|c|c|c|c|c|c|c|c|c|}
\hline \multicolumn{10}{|c|}{ Four Methods Comparison with 4 FUs } \\
\hline \multirow[t]{2}{*}{ Bench. } & \multirow[t]{2}{*}{$\mathrm{N}$. } & M1 & M2 & \multicolumn{3}{|c|}{ M3 } & \multicolumn{3}{|c|}{ M4 } \\
\hline & & $\begin{array}{c}E \\
(\mu J)\end{array}$ & $\begin{array}{c}\mathrm{E} \\
(\mu \mathrm{J})\end{array}$ & $\begin{array}{c}E \\
(\mu J)\end{array}$ & $\begin{array}{c}\% \text { M1 } \\
(\%)\end{array}$ & $\begin{array}{c}\% \text { M2 } \\
(\%)\end{array}$ & $\begin{array}{c}\mathrm{E} \\
(\mu \mathrm{J})\end{array}$ & $\begin{array}{c}\% \text { M1 } \\
(\%)\end{array}$ & $\begin{array}{c}\% \mathrm{M} 2 \\
(\%)\end{array}$ \\
\hline WDF(1) & 4 & 768 & 665 & 534 & 30.5 & 19.7 & 505 & 34.2 & 24.1 \\
\hline WDF(2) & 12 & 2298 & 2006 & 1603 & 30.2 & 20.1 & 1521 & 33.8 & 24.2 \\
\hline IIR & 16 & 3041 & 2601 & 2104 & 30.8 & 19.1 & 1979 & 34.9 & 23.9 \\
\hline DPCM & 16 & 3102 & 2669 & 2168 & 30.1 & 18.8 & 2021 & 34.8 & 24.3 \\
\hline $2 \mathrm{D}(1)$ & 34 & 6523 & 5645 & 4521 & 30.7 & 19.9 & 4294 & 34.2 & 23.9 \\
\hline $2 \mathrm{D}(2)$ & 4 & 812 & 705 & 569 & 29.9 & 19.3 & 531 & 34.6 & 24.7 \\
\hline MDFG1 & 8 & 1687 & 1483 & 1185 & 29.8 & 20.1 & 1121 & 33.6 & 24.4 \\
\hline MDFG2 & 8 & 1811 & 1568 & 1273 & 29.7 & 18.8 & 1187 & 34.5 & 24.3 \\
\hline Floyd & 16 & 3257 & 2822 & 2265 & 30.5 & 19.7 & 2145 & 34.1 & 24.0 \\
\hline All-pole & 29 & 5503 & 4790 & 3831 & 30.4 & 20.0 & 3623 & 34.2 & 24.4 \\
\hline & era & Reduc & n (\%) & & 30.3 & 19.6 & & 34.3 & 24.2 \\
\hline
\end{tabular}

TABLE III

THE COMPARISON OF TOTAL FAILURE RATE WITH FOUR METHODS ON VARIOUS BENCHMARKS WHEN THE TIME CONSTRAINT IS 1000.

rate is minimized. A node can only be rescheduled to a FU if its finish time is earlier than that in ALAP and the task does not overlap with other tasks remaining in the ALAP schedule. The choice of the node is made this way because the remaining nodes can always be scheduled within the time constraint as long as the ALAP schedule exists for this task graph. After this task is scheduled, mark the node, update the dependence constraint information and the earliest start time for all its descendants recursively, update the system failure rate and the schedule length, then continue this process until all nodes are marked.

\section{EXPERIMENTS}

In this section, we present our experiments with the our algorithms on a set of well-known DSP benchmarks including Wave Digital filter (WDF), Infinite Impulse filter (IIR), Differential Pulse-Code Modulation device (DPCM), Two dimensional filter (2D), Floyd-Steinberg algorithm (Floyd), and All-pole filter. We build a simulation framework to evaluate the effectiveness of our approach. $\mathrm{K}$ different $\mathrm{FU}$ types, $F_{1}, \cdots, F_{K}$, are used in the system, in which a FU with type $F_{1}$ is the quickest with the highest failure rate and a FU with type $F_{K}$ is the slowest with the lowest failure rate. Each task node has different failure rates under different FU types. Due to the page limit, we don't list the detail table in this paper.

We conducted experiments on four methods: Method 1: list scheduling; Method 2: the TBFT algorithm in [9]; Method 3: our algorithm FU_Sch1; Method 4: our algorithm FU_Sch2. In the list scheduling, the priority of a node is set as the longest path from this node to a leaf node [12]. The experiments are performed on a Dell PC with a P4 $2.1 \mathrm{G}$ processor and 512 MB memory running Red Hat Linux 9.0.

The experimental results for the four methods are shown in Table II to Table III when the number of FUs is 3 and 4, respectively. Column "Bench." stands for the benchmarks we used in the experiments. Column "N." represents the number of nodes of each filter benchmark. Column "M1" to "M4" represents the four methods we used in the experiments. Column "E" represents the minimum total system failure rate obtained from four different algorithms. Column "\% M1", "\% M2" under "M3" and "M4" represents the percentage of reduction in total failure rate, compared to Method 1 and Method 2, respectively. The average reduction 


\begin{tabular}{|c||c||c||c||c|c||c|c|c|}
\hline \multicolumn{9}{|c|}{ Four Methods Comparison on IIR with 3 FUs } \\
\hline \multirow{2}{*}{ Time } & M1 & M2 & \multicolumn{3}{c|}{ M3 } & \multicolumn{3}{c|}{ M4 } \\
\cline { 2 - 8 } & E & E & E & M1 & $\%$ M2 & E & $\%$ M1 & $\%$ M2 \\
$(\mu \mathrm{J})$ & $(\mu \mathrm{J})$ & $(\mu \mathrm{J})$ & $(\%)$ & $(\%)$ & $(\mu)$ & $(\%)$ \\
\hline 300 & 2702 & 2630 & 2329 & 13.8 & 11.4 & 2256 & 16.5 & 14.2 \\
400 & 2650 & 2578 & 2221 & 16.2 & 13.9 & 2176 & 17.9 & 15.6 \\
500 & 2627 & 2473 & 2136 & 18.7 & 13.6 & 2067 & 21.3 & 16.4 \\
600 & 2603 & 2356 & 2017 & 22.5 & 14.4 & 1937 & 25.6 & 17.8 \\
700 & 2578 & 2302 & 1939 & 24.8 & 15.8 & 1872 & 27.4 & 18.7 \\
800 & 2527 & 2222 & 1867 & 26.1 & 16.0 & 1787 & 29.3 & 19.6 \\
900 & 2478 & 2148 & 1802 & 27.3 & 16.1 & 1712 & 30.9 & 20.3 \\
1000 & 2456 & 2135 & 1751 & 28.7 & 18.0 & 1678 & 31.7 & 21.4 \\
1100 & 2431 & 2106 & 1707 & 29.8 & 19.0 & 1641 & 32.5 & 22.1 \\
1200 & 2381 & 2046 & 1655 & 30.5 & 19.1 & 1568 & 33.5 & 22.5 \\
\hline
\end{tabular}

TABLE IV

THE COMPARISON OF TOTAL FAILURE RATE WITH FOUR METHODS ON IIR FILTER UNDER DIFFERENT TIME CONSTRAINTS.

is shown in the last row of the table.

The results show that our algorithms for the FRMFS problem can significantly improve the performance of applications on WSNs. All of our algorithms (Method 3 and Method 4) improve the failure-rate reduction over the traditional list scheduling algorithm. Among them, FU_Sch2 (Method 4) gives the best performance. We can see that with more FUs selections, the reduction ratio for the total failure rate has increased. For example, with 3 FUs, compared with Method 1, FU_Sch2 (Method 4) shows an average $31.1 \%$ reduction in total failure rate. While using 4 FUs, the reduction rate changed to be $34.3 \%$ for total failure rate.

It is worthwhile to pointing out that we obtain this improvement ratio without sacrificing performance. For example, Table IV shows the failure rate vs. time constraints by benchmark IIR filter. FU_Sch2 (Method 4) improves the failure-rate reduction significantly when the time constraint is large. If the time constraint is small, it still improves the failure-rate reduction while meeting the constraint. FU_Sch1 (Method 3) can always improve failure-rate reduction as long as there exists a schedule by the ALAP scheduling. The improvement is not as significant as FU_Sch2 (Method 4) when the time constraint is large. This is because the algorithm always tries to use all available FUs in each step, while FU_Sch2 (Method 4) schedules one node in each step and avoid FUs with high failure rate if possible. Both FU_Sch1 and FU_Sch2 are novel algorithms. FU_Sch1 is more straight forward and has lower complexity. FU_Sch2 can achieve better results but is more suitable for smaller systems. Both these two algorithms can give solutions that are very close to the optimal solutions (less than $15 \%$ to $5 \%$ difference in average) in most cases.

\section{CONCLUSION}

In this paper, we studied the scheduling problem that minimizes the total failure rate of a system without sacrificing performance on WSNs. We proposed two highly efficient algorithms to solve the FRMFS (Failure Rate Minimization with FU Scheduling) problem for WSN applications. By using multi-FU scheduling, our algorithms improve both the performance and failure-rate-minimization of WSN applications. A wide range of benchmarks have been tested on the experiments. The experimental results showed that our algorithms significantly improved the failure-rate-minimization while satisfying performance requirements for applications on WSNs.

\section{REFERENCES}

[1] G. Toll, J. Polastre, R. Szewczyk, D. Culler, N. Turner, K. Tu, and et al., "A macroscope in the redwoods," in ACM SenSys'05, San Diego, CA, USA, Nov. 2-4, 2005, pp. 51-63.

[2] G. Werner, P. Swieskowski, and M. Welsh, "Demonstration: real-time volcanic earthquake localization," in ACM SenSys'06, Boulder, CO, USA, Nov. 1-3, 2006, pp. 357-358.

[3] L. Laffea, R. Monson, R. Han, R. Manning, A. Glasser, S. Oncley, and et al., "Comprehensive monitoring of $\mathrm{CO} 2$ sequestration in subalpine forest ecosystems and its relation to global warming," in ACM SenSys'06, Boulder, CO, USA, Nov. 1-3, 2006, pp. 423-424.

[4] R. Szewczyk, E. Osterweil, J. Polastre, M. Hamilton, A. Mainwaring, and D. Estrinet, "Habitat monitoring with sensor networks," Соттиnications of ACM, vol. 47, no. 6, pp. 34-40, 2004.

[5] P. Zhang, C. M. Sadler, S. Lyon, and M. Martonosi, "Hardware design experiences in zebranet," in ACM SenSys'04, Baltimore, Maryland, USA, Nov. 3-5, 2004.

[6] P. Sikka, P. Corke, P. Valencia, C. Crossman, D. Swain, and G. B.Hurley, "Wireless adhoc sensor and actuator networks on the farm," in ACM SenSys'06, Boulder, CO, USA, Nov. 1-3, 2006, pp. 492-499.

[7] D. S. Glaser, "Some real-world applications of wireless sensor nodes," in SPIE Symposium on smart structures and materials, San Diego, CA, USA, Mar. 2004.

[8] S. B. Eisenman and A. T. Campbell, "Poster abstract: skiscape sensing," in ACM SenSys'06, Boulder, CO, USA, Nov. 1-3, 2006, pp. 401-402.

[9] S. Srinivasan and N. K. Jha, "Safety and reliability driven task allocation in distributed systems," IEEE Trans. on Parallel and Distributed Systems, vol. 10, pp. 238-251, Mar. 1999.

[10] S. Borkar, N. Jouppi, and P. Stenstrom, "Microprocessors in the era of terascale integration," in IEEE/ACM DATE, April 2007, pp. 1-6.

[11] S. M. Shatz, J.-P. Wang, and M. Goto, "Task allocation for maximizing reliability of distributed computer systems," IEEE Trans. on Computers, vol. 41, pp. 1156-1168, Sep. 1992.

[12] G. D. Micheli, Synthesis and Optimization of Digital Circuits. McGraw-Hill, 1994. 